\title{
NOTE ON CUP-PRODUCTS
}

I. M. JAMES

1. Introduction. We consider $C W$-complexes, $K$, of the form $e^{n+q} \cup e^{n} \cup S^{q}$, where $n-1>q \geqq 2$. That is to say, we regard the $q$ sphere, $S^{q}$, as consisting of a 0 -cell and its complementary $q$-cell, we attach an $n$-cell $e^{n}$ to $S^{q}$, and then we attach an $(n+q)$-cell $e^{n+q}$ to $e^{n} \cup S^{q}$. Consider the cohomology of $K$ with coefficients in the ring of integers. Let $x, y, z$ denote the cohomology classes which are carried by $e^{n}, S^{q}, e^{n+q}$, respectively. I define the characteristic of $K$ to be the integer, $m$, such that

$$
x \cup y=m z .
$$

For example, $m= \pm 1$ in a manifold. Let $\alpha \in \pi_{n-1}\left(S^{q}\right)$ denote the homotopy class of the map $S^{n-1} \rightarrow S^{q}$ by which $e^{n}$ is attached. Then I describe $K$ as a complex of type $(m, \alpha)$. Our main result is:

TheOREM (1.2). Let $\alpha \in \pi_{n-1}\left(S^{q}\right)$, where $n-1>q \geqq 2$. Let $\left[\alpha, i_{q}\right]$ $\in \pi_{n+q-2}\left(S^{q}\right)$ denote the Whitehead product ${ }^{1}$ of $\alpha$ and a generator $i_{q} \in \pi_{q}\left(S^{q}\right)$. Then there exists a complex $e^{n+q} \cup e^{n} \cup S^{q}$ of type $(m, \alpha)$ if, and only if, $m\left[\alpha, i_{q}\right]$ is contained in the image of the homomorphism

$$
\alpha_{*}: \pi_{n+q-2}\left(S^{n-1}\right) \rightarrow \pi_{n+q-2}\left(S^{q}\right),
$$

which is induced by $\alpha$.

This theorem has significant applications from two points of view. On the one hand, the relations discovered by Adem $[1 ; 2]$, between the Steenrod cohomology operations lead to new relations for the Whitehead product. On the other hand, results from the theory of Whitehead products lead to realization theorems for the cohomology ring. For example, we deduce:

Corollary (1.3). There exists a complex $e^{n+q} \cup e^{n} \cup S^{q}$ of type $(m, \alpha)$ if, and only if, $m$ is a multiple of $k$, where $k$ is a positive integer determined by $\alpha$.

For since $n-1>q \geqq 2$, either $\pi_{n-1}\left(S^{q}\right)$ or $\pi_{n+q-2}\left(S^{q}\right)$ is a finite group, by Proposition 5 on p. 498 of [11]. Therefore $\left[\alpha, i_{q}\right]$ has finite order, and hence the projection of $\left[\alpha, i_{q}\right]$ has order $k$, say, in the factor

Received by the editors April 14, 1956.

1 This is the notation for the product defined by J. H. C. Whitehead in [14]. (Numbers in square brackets refer to the articles listed at the end of the present note.) 
group of $\pi_{n+q-2}\left(S^{q}\right)$ by the image of $\alpha_{*}$. Hence (1.3) follows from (1.2). In particular we have

Corollary (1.4). If $\left[\alpha, i_{q}\right]=0$, then there exists a complex of type $(m, \alpha)$ for arbitrary $m$. This is the case, for example, if $q=2,3$, or $7, b y$ (2.2) and (2.4) of [5].

By (4.5) of [7], a Whitehead product in the homotopy groups of an odd-dimensional sphere is either zero or of order two. Hence we have

Corollary (1.5). Let $\alpha \in \pi_{n-1}\left(S^{q}\right)$, where $q$ is odd and $n-1>q \geqq 3$. Then there exists a complex $e^{n+q} \cup e^{n} \cup S^{q}$ of type $(m, \alpha)$ for arbitrary even integer $m$.

The most interesting case is when $n=2 q$ and $q$ is even, so that $K$ has the form $e^{3 q} \cup e^{2 q} \cup S^{q}$. Let $x, y, z$ mean the same as before. By (4.1) of [4] we have

$$
y \cup y=h x,
$$

where $h$ denotes the Hopf invariant of $\alpha$. Hence, by (1.1),

$$
y \cup y \cup y=h m z \text {. }
$$

We pass to cohomology in the ring of integers, mod 3. Let $x^{\prime}, y^{\prime}, z^{\prime}$ denote the classes $x, y, z$, respectively, reduced mod 3 . Consider the homomorphism

$$
\rho^{q / 2}: H^{q}\left(K ; Z_{3}\right) \rightarrow H^{3 q}\left(K ; Z_{3}\right),
$$

which is defined by Steenrod in [13]. We have $\rho^{q / 2}\left(y^{\prime}\right)=\left(y^{\prime}\right)^{3}$, by (6.10) of [13]; and hence $\rho^{q / 2}\left(y^{\prime}\right)=h m z^{\prime}$, by (1.7). But since $K$ has trivial cohomology except in dimensions $q, 2 q$, and $3 q$, it follows from the Adem basis theorem $((1.4)$ of $[2])$ that $\beta^{q / 2}\left(y^{\prime}\right)=0$ unless $q / 2$ or $q / 4$ is equal to an integral power of three. Hence we obtain:

TheOREM (1.8). Let $\alpha \in \pi_{2 q-1}\left(S^{q}\right)$ be an element of Hopf invariant prime to three, where $q$ is even. Suppose that neither $q / 2$ nor $q / 4$ is equal to an integral power of three. Then there exists no complex $e^{3 q} \cup e^{2 q} \cup S^{q}$ of type ( $m, \alpha)$ unless $m$ is divisible by three. Hence, by (1.2), there exists no element $\beta \in \pi_{3 q-2}\left(S^{2 q-1}\right)$ such that ${ }^{2} m\left[\alpha, i_{q}\right]=\alpha \circ \beta$ unless $m$ is divisible by three.

In [10], Nakaoka and Toda prove a special case of (1.8) and use it to show that the triple Whitehead product $\left[\left[i_{q}, i_{q}\right], i_{q}\right]$ is of order three unless $q / 2$ is equal to an integral power of three. In [8], I use the following corollary of (1.8) to prove that the sphere $S^{q-1}$ cannot

2 The small circle, centred, denotes composition. 
carry a homotopy-associative multiplication unless $q=2$ or 4 .

By (4.1) of [1], $\pi_{2 q-1}\left(S^{q}\right)$ contains no elements of odd Hopf invariant unless $q$ is equal to an integral power of two. Hence we obtain from (1.8):

Corollary (1.9). Let $\alpha \in \pi_{2 q-1}\left(S^{q}\right)$, where $q \geqq 6$. Suppose that $h \equiv \pm 1, \bmod 6$, where $h$ is the Hopf invariant of $\alpha$. Then there exists no complex $e^{8 q} \cup e^{2 q} \cup S^{q}$ of type $(m, \alpha)$ unless $m$ is divisible by three. Hence, by (1.2), there exists no element $\beta \in \pi_{3 q-2}\left(S^{2 q-1}\right)$ such that $m\left[\alpha, i_{q}\right]=\alpha \circ \beta$ unless $m$ is divisible by three.

Apart from these applications, the characteristic of the complex $e^{n+q} \cup e^{n} \cup S^{q}$ plays an important rôle in the determination of its homotopy groups. I hope to develop this point in a second note. It is worth remarking that the present topic can also be discussed in terms of the cohomology invariants of mappings which are defined in [12].

2. The relative Whitehead product. Let $S^{r-1}$ denote the unit sphere in $r$-dimensional euclidean space, where $r \geqq 2$, and let $V^{r}$ denote the convex hull of $S^{r-1}$. Let $X$ be a space, and let $A$ be a nonvoid subspace. The elements of the relative homotopy group $\pi_{r}(X, A)$ are homotopy classes of maps ${ }^{3}$ of $\left(V^{r}, S^{r-1}\right)$ into $(X, A)$, and the elements of the absolute homotopy group $\pi_{r-1}(A)$ are homotopy classes of maps of $S^{r-1}$ into $A$. The boundary operator $\partial: \pi_{r}(X, A) \rightarrow \pi_{r-1}(A)$ is defined by restricting representatives. We denote by $i_{r}$ the element of $\pi_{r}\left(S^{r}\right)$ which is represented by the identity map of $S^{r}$. We also represent $i_{r}$ by a map of $V^{r}$ onto $S^{r}$, which is constant on $S^{r-1}$, so that the injection homomorphism $i_{*}: \pi_{r}(X) \rightarrow \pi_{r}(X, A)$ is defined by composing representatives with this map.

The Whitehead product, as defined in [14], is a natural bilinear pairing of $\pi_{p}(X)$ with $\pi_{q}(X)$ to $\pi_{p+q-1}(X)$, where $p, q \geqq 2$. The product of $\lambda \in \pi_{p}(X)$ and $\mu \in \pi_{q}(X)$ is denoted by $[\lambda, \mu]$. The relative Whitehead product, as defined in [3], is a natural bilinear pairing of $\pi_{p}(X, A)$ with $\pi_{q}(A)$ to $\pi_{p+q-1}(X, A)$, where $p \geqq 3, q \geqq 2$. The product of $\lambda^{\prime} \in \pi_{p}(X, A)$ and $\mu^{\prime} \in \pi_{q}(A)$ is denoted by $\left[\lambda^{\prime}, \mu^{\prime}\right]$. There are two relations between the relative product and the ordinary one. First, by (3.5) of [3] we have

$$
\partial\left[\lambda^{\prime}, \mu^{\prime}\right]+\left[\partial\left(\lambda^{\prime}\right), \mu^{\prime}\right]=0 .
$$

Secondly, let $\mu$ denote the injection of $\mu^{\prime}$ into $\pi_{q}(X)$, and suppose that $\lambda^{\prime}=i_{*}(\lambda)$, where $\lambda \in \pi_{p}(X)$. Then we have

The usual restrictions involving basepoints are to be understood throughout. 


$$
i_{*}[\lambda, \mu]=\left[\lambda^{\prime}, \mu^{\prime}\right],
$$

by (3.3) and (3.10) of [3].

3. The main theorem. Let $\alpha \in \pi_{n-1}\left(S^{q}\right)$, where $n-1>q \geqq 2$. Let $L$ be the complex which is obtained by attaching an $n$-cell $e^{n}$ to $S^{q}$ by a map of homotopy class $\alpha$. Let $\sigma \in \pi_{n}\left(L, S^{q}\right)$ denote the homotopy class of the attaching map of $e^{n}$, so that $\alpha=\partial(\sigma)$. Let

$$
\sigma_{*}: \pi_{n+q-1}\left(V^{n}, S^{n-1}\right) \rightarrow \pi_{n+q-1}\left(L, S^{q}\right)
$$

denote the homomorphism which is induced by $\sigma$. By (1.4) of [6], we have that $\sigma_{*}$ is an isomorphism into, and that $\pi_{n+q-1}\left(L, S^{q}\right)$ is the direct sum of (i) the image of $\sigma_{*}$ and (ii) an infinite cyclic subgroup generated by the relative Whitehead product $\left[\sigma, i_{q}\right]$. In other words, a given element $\gamma \in \pi_{n+q-1}\left(L, S^{q}\right)$ has a unique expression:

$$
\gamma=m\left[\sigma, i_{q}\right]+\sigma \circ \rho,
$$

where $m$ is an integer, and $\rho \in \pi_{n+q-1}\left(V^{n}, S^{n-1}\right)$. By $(2.1),-\partial\left[\sigma, i_{q}\right]$ $=\left[\partial(\sigma), i_{q}\right]=\left[\alpha, i_{q}\right]$, and by the naturality of the boundary operator, $\partial(\sigma \circ \rho)=\partial(\sigma) \circ \partial(\rho)=\alpha \circ \tau$, where $\tau=\partial(\rho)$. Therefore

$$
\partial(\gamma)=-m\left[\alpha, i_{q}\right]+\alpha \circ \tau \text {. }
$$

Consider the following portion from the homotopy exact sequence of the pair $\left(L, S^{q}\right)$ :

$$
\pi_{r}(L) \stackrel{i_{*}}{\rightarrow} \pi_{r}\left(L, S^{q}\right) \stackrel{\partial}{\rightarrow} \pi_{r-1}\left(S^{q}\right) .
$$

The remainder of this note is devoted to proving:

Theorem (3.3). Let $\beta \in \pi_{n+q-1}(L)$, and let $K$ denote the complex which is obtained by attaching an $(n+q)$-cell to $L$ by a map of homotopv class $\beta$. Then

$$
i_{*}(\beta)=m\left[\sigma, i_{q}\right]+\sigma \circ \rho,
$$

where $m$ is the characteristic of $K$, and $\rho \in \pi_{n+q-1}\left(V^{n}, S^{n-1}\right)$.

Assuming (3.3), the proof of (1.2) is completed as follows. Let $m$ be the characteristic of $K$, as in (3.3). Then $\partial i_{*}(\beta)=0$, by exactness, and so $m\left[\alpha, i_{q}\right]=\alpha \circ \tau$, by (3.2), where $\tau=\partial(\rho)$. Therefore $m\left[\alpha, i_{q}\right]$ lies in the image of the homomorphism

$$
\alpha_{*}: \pi_{n+q-2}\left(S^{n-1}\right) \rightarrow \pi_{n+q-2}\left(S^{q}\right) .
$$

Conversely, suppose that $m\left[\alpha, i_{q}\right]$ lies in the image of $\alpha_{*}$, for some integer $m$. Then there is an element $\tau \in \pi_{n+q-2}\left(S^{n-1}\right)$ such that $m\left[\alpha, i_{q}\right]=\alpha \circ \tau$. Consider the element 


$$
\gamma=m\left[\sigma, i_{q}\right]+\sigma \circ \rho,
$$

where $\rho$ is the element of $\pi_{n+q-1}\left(V^{n}, S^{n-1}\right)$ such that $\partial(\rho)=\tau$. We have $\partial(\gamma)=0$, by (3.2). Hence, by exactness, there is an element $\beta \in \pi_{n+q-1}(L)$ such that $i_{*}(\beta)=\gamma$. Let $K$ be the complex which is obtained by attaching an $(n+q)$-cell to $L$ by a map of homotopy class $\beta$. Then the characteristic of $K$ is equal to $m$, by (3.3). This completes the proof of (1.2) subject, of course, to proving (3.3).

4. A formula for cup-products. The cohomology theory in what follows has coefficients in the ring of integers. Consider a $C W$ complex $X$, and a pair of elements $a \in H^{p}(X), b \in H^{q}(X)$, such that $a \cup b=0$, where $p, q \geqq 2$. Suppose that $H^{p+q-1}(X)$ is finite. Then I define an integer-valued function, $h$, on $\pi_{p+q-1}(X)$ as follows. Let $\lambda \in \pi_{p+q-1}(X)$. Let $X^{*}$ denote the complex which is obtained by attaching an oriented $(p+q)$-cell to $X$ by a map of homotopy class $\lambda$. Let $c$ denote the cohomology class which is carried by the cell. There are unique elements $a^{\prime} \in H^{p}\left(X^{*}\right), b^{\prime} \in H^{q}\left(X^{*}\right)$, which map into $a, b$, respectively, under the injection. Since $a \cup b=0$ in $X$, there is an integer $m$ such that $a^{\prime} \cup b^{\prime}=m c$. I define $h(\lambda)=m$. We prove:

THEOREM (4.1). The function $h$ constitutes a homomorphism of $\pi_{p+q-1}(X)$ into the group of integers.

We prove (4.1) in the following form:

Lemma (4.2). Let $\lambda_{1}, \lambda_{2}, \lambda_{3} \in \pi_{p+q-1}(X)$ be elements such that $\lambda_{1}+\lambda_{2}$ $+\lambda_{3}=0$. Then

$$
h\left(\lambda_{1}\right)+h\left(\lambda_{2}\right)+h\left(\lambda_{3}\right)=0 .
$$

In what follows, let $t$ be an indexing integer which takes values $1,2,3$. Let $X_{t}$ denote the complex which is obtained by attaching a $(p+q)$-cell $e_{t}$ to $X$ by a map of homotopy class $\lambda_{t}$, so that

$$
X_{2} \cap X_{3}=X_{1} \cap X_{3}=X_{1} \cap X_{2}=X .
$$

Let $X^{\prime}=X_{1} \cup X_{2} \cup X_{3}$. Consider the injections:

$$
H^{r}\left(X^{\prime}\right) \underset{j_{t}}{\rightarrow} H^{r}\left(X_{t}\right) \underset{i_{t}}{\rightarrow} H^{r}(X) .
$$

Let $a^{\prime}, b^{\prime}$ denote the cohomology classes of $X^{\prime}$ such that $i_{t} j_{t}\left(a^{\prime}\right)=a$, $i_{t} j_{t}\left(b^{\prime}\right)=b$, and let $c_{t}$ denote the class which is carried by $e_{t}$. Since $a \cup b=0$ in $X$, there are integers $m_{t}$ such that

$$
a^{\prime} \cup b^{\prime}=m_{1} c_{1}+m_{2} c_{2}+m_{3} c_{3} .
$$

However, by the naturality of the cup-product, 


$$
\begin{aligned}
i_{t}\left(a^{\prime}\right) \cup j_{t}\left(b^{\prime}\right) & =j_{t}\left(a^{\prime} \cup b^{\prime}\right) \\
& =m_{1} j_{t}\left(c_{1}\right)+m_{2} j_{t}\left(c_{2}\right)+m_{3} j_{t}\left(c_{3}\right) \\
& =m_{t} j_{t}\left(c_{t}\right) .
\end{aligned}
$$

Hence $m_{t}=h\left(\lambda_{t}\right)$, by the definition of $h$. Since $\lambda_{1}+\lambda_{2}+\lambda_{3}=0$, by hypothesis, there is a map $f: S^{p+q} \rightarrow X^{\prime}$ which maps $S^{p+q}$ onto each of the three cells $e_{t}$ with degree 1 . Let $f^{*}: H^{r}\left(X^{\prime}\right) \rightarrow H^{r}\left(S^{p+q}\right)$ denote the homomorphism induced by $f$, which is trivial unless $r=p+q$. Then $f^{*}\left(c_{t}\right)=c$, the cohomology class of $S^{p+q}$. Hence

$$
\begin{aligned}
\left(m_{1}+m_{2}+m_{3}\right) c & =f^{*}\left(m_{1} c_{1}+m_{2} c_{2}+m_{3} c_{3}\right) \\
& =f^{*}\left(a^{\prime} \cup b^{\prime}\right) \\
& =f^{*}\left(a^{\prime}\right) \cup f^{*}\left(b^{\prime}\right),
\end{aligned}
$$

which is zero. Therefore $m_{1}+m_{2}+m_{3}=0$, since $H^{p+q}\left(S^{p+q}\right)$ is freely generated by $c$. Since $m_{t}=h\left(\lambda_{t}\right)$, this proves (4.2). The passage from (4.2) to (4.1) is elementary, and will be omitted.

It is worth mentioning that $h(\lambda)$ can equally well be defined to be the functional cup-product [12] of $a$ and $b$ with respect to $\lambda$.

5. Analysis of the homotopy groups. We make preparations for the proof of (3.3), which will be completed in the next section. Let the notation mean the same as in $\S 3$. Thus $\alpha \in \pi_{n-1}\left(S^{q}\right)$, where $n-1>q$ $\geqq 2$, and $L$ is the complex which is obtained by attaching $e^{n}$ to $S^{a}$ by a map of homotopy class $\alpha$. The characteristic map of $e^{n}$ represents a generator $\sigma \in \pi_{n}\left(L, S^{q}\right)$, such that $\partial(\sigma)=\alpha$. We now attach $S^{n}$ to $L$ at $x_{0}$, the 0 -cell in $S^{q}$, so that $S^{n} \cap L=x_{0}$. Let $M=S^{n} \cup L$. Consider the following maps, where $u$ is the inclusion map, $v$ is the retraction such that $v S^{n}=x_{0}$, and $g$ is a map such that (i) $g$ maps $S^{q}$ identically, (ii) $v \circ g \simeq 1$, and (iii) $g$ maps $\bar{e}^{n}$ onto $S^{n}$ with degree 1 :

$$
\left(L, S^{q}\right) \stackrel{g}{\rightarrow}\left(M, S^{q}\right) \stackrel{v}{\rightarrow}\left(L, S^{q}\right) \stackrel{u}{\rightarrow}\left(M, S^{q}\right) .
$$

Let $g_{*}, g_{*}$, etc. denote the homomorphisms induced by $g$, etc., as shown in the following commutative diagram together with the injections $i_{*}$ and $j_{*}$.

$$
\begin{aligned}
& \pi_{r}(L) \stackrel{g_{*}}{\rightarrow} \pi_{r}(M) \stackrel{v_{*}}{\longrightarrow} \pi_{r}(L) \stackrel{u_{*}}{\longrightarrow} \pi_{r}(M) \\
& i_{* \downarrow} \quad j_{*} \downarrow \quad i_{*} \downarrow \quad j_{*} \downarrow \\
& \pi_{r}\left(L, S^{q}\right) \underset{g_{f}}{\rightarrow} \pi_{r}\left(M, S^{q}\right) \underset{v_{f}}{\rightarrow} \pi_{r}\left(L, S^{q}\right) \underset{u_{f}}{\rightarrow} \pi_{r}\left(M, S^{q}\right) .
\end{aligned}
$$

Since $v \circ u=1$ and $v \circ g \simeq 1$, we have at once: 


$$
v_{*} u_{*}=1, \quad v_{*} g_{*}=1 .
$$

Let $\kappa_{n} \in \pi_{n}(M)$ denote the homotopy class of the inclusion map $S^{n} \rightarrow M$, and let $\lambda_{n}=j_{*}\left(\kappa_{n}\right) \in \pi_{n}\left(M, S^{q}\right)$. Let $\rho \in \pi_{n+q-1}\left(V^{n}, S^{n-1}\right)$, so that $\lambda_{n} \circ \rho \in \pi_{n+q-1}\left(M, S^{q}\right)$. I assert that:

$$
\text { (a) }\left\{\begin{array}{l}
g_{\sharp}\left[\sigma, i_{q}\right]=u_{\sharp}\left[\sigma, i_{q}\right]+\left[\lambda_{n}, i_{q}\right], \\
g_{\sharp}(\sigma \circ \rho)=u_{\sharp}(\sigma \circ \rho)+\lambda_{n} \circ \rho .
\end{array}\right.
$$

For it follows from the definition of $g$ that $g \sharp(\sigma)=u \sharp(\sigma)+\lambda_{n}$. Hence

$$
\begin{aligned}
g_{\sharp}\left[\sigma, i_{q}\right] & =\left[g_{\sharp}(\sigma), i_{q}\right], \text { bv naturality, } \\
& =\left[u_{\sharp}(\sigma), i_{q}\right]+\left[\lambda_{n}, i_{q}\right], \text { by linearity, } \\
& =u_{\sharp}\left[\sigma, i_{q}\right]+\left[\lambda_{n}, i_{q}\right], \text { by naturality. }
\end{aligned}
$$

This proves (a). In the case of (b), we have

$$
\begin{aligned}
g_{\sharp}(\sigma \circ \rho) & =g_{\sharp}(\sigma) \circ \rho=\left(u \sharp(\sigma)+\lambda_{n}\right) \circ \rho \\
& =u_{\sharp}(\sigma) \circ \rho+\lambda_{n} \circ \rho, \text { by }(23.13) \text { of }[9], \\
& =u_{\sharp}(\sigma \circ \rho)+\lambda_{n} \circ \rho .
\end{aligned}
$$

This completes the proof of (5.3).

We use these relations to prove

Lemma (5.4). Let $\beta \in \pi_{n+q-1}(L)$ be an element such that

$$
i_{*}(\beta)=m\left[\sigma, i_{q}\right]+\sigma \circ \rho,
$$

where $m$ is an integer and $\rho \in \pi_{n+q-1}\left(V^{n}, S^{n-1}\right)$. Let w denote the Whitehead product $\left[\kappa_{n}, \kappa_{q}\right] \in \pi_{n+q-1}(M)$, where $\kappa_{r} \in \pi_{r}(M)(r=n, q)$ denotes the homotopy class of the inclusion map $S^{r} \rightarrow M$. Then

$$
g_{*}(\beta)=u_{*}(\beta)+m w+\kappa_{n} \circ \rho .
$$

We have to show that the element

$$
\theta=u_{*}(\beta)+m w+\kappa_{n} \circ \rho-g_{*}(\beta)
$$

is zero. We first of all check that:

$$
\text { (a) }\left\{\begin{array}{l}
j_{*}(\theta)=0, \\
v_{*}(\theta)=0 .
\end{array}\right.
$$

In the case of (a), we have

$$
\begin{aligned}
g_{\sharp} i_{*}(\beta) & =m g_{\sharp}\left[\sigma, i_{q}\right]+g_{\sharp}(\sigma \circ \rho) \\
& =m u_{\sharp}\left[\sigma, i_{q}\right]+m\left[\lambda_{n}, i_{q}\right]+u_{\sharp}(\sigma \circ \rho)+\lambda_{n} \circ \rho, \text { by }(5.3), \\
& =u_{\sharp} i_{*}(\beta)+m\left[\lambda_{n}, i_{q}\right]+\lambda_{n} \circ \rho .
\end{aligned}
$$


However, since (5.1) is commutative we have

$$
g_{*} i_{*}(\beta)-u_{\sharp} i_{*}(\beta)=j_{*} g_{*}(\beta)-j_{*} u_{*}(\beta),
$$

and therefore

$$
j_{*} g_{*}(\beta)=j_{*} u_{*}(\beta)+m\left[\lambda_{n}, i_{q}\right]+\lambda_{n} \circ \rho .
$$

However, $\left[\lambda_{n}, i_{q}\right]=\left[j_{*}\left(\kappa_{n}\right), i_{q}\right]=j_{*}\left[\kappa_{n}, \kappa_{q}\right]$, by (2.2), and $\lambda_{n} \circ \rho$ $=j_{*}\left(\kappa_{n}\right) \circ \rho=j_{*}\left(\kappa_{n} \circ \rho\right)$. Therefore

$$
j_{*} g_{*}(\beta)=j_{*} u_{*}(\beta)+m j_{*}(w)+j_{*}\left(\kappa_{n} \circ \rho\right) .
$$

This proves (a). In the case of (b), we have $v_{*}\left(\kappa_{n}\right)=0$, since $v S^{n}=x_{0}$. Therefore $v_{*}\left(\kappa_{n} \circ \rho\right)=v_{*}\left(\kappa_{n}\right) \circ \rho=0$, and $v_{*}(w)=v_{*}\left[\kappa_{n}, \kappa_{q}\right]=0$, by naturality. Moreover, $v_{*} u_{*}(\beta)=v_{*} g_{*}(\beta)$, by (5.2). Hence $v_{*}(\theta)=0$. which completes the proof of (5.5).

Since $j_{*}(\theta)=0$, it follows from the exactness of the homotopy sequence of the pair $(M, L)$ that $\theta=u_{*}(\phi)$, where $\phi \in \pi_{n+q-1}(L)$. Therefore

$$
\begin{aligned}
\theta & =u_{*} v_{*} u_{*}(\phi), \text { by }(5.2), \\
& =u_{*} v_{*}(\theta)=0
\end{aligned}
$$

by (5.5b). This completes the proof of (5.4).

6. Proof of the main theorem. We carry straight on from the previous section so as to prove (3.3) and thereby complete the proof of (1.2). Thus $L=e^{n} \cup S^{q}$, and $\beta \in \pi_{n+q-1}(L)$ is an element such that

$$
i_{*}(\beta)=m\left[\sigma, i_{q}\right]+\sigma \circ \rho,
$$

where $m$ is an integer and $\rho \in \pi_{n+q-1}\left(V^{n}, S^{n-1}\right)$, as in (3.3). Let $K$ be the complex which is obtained by attaching $e^{n+q}$ to $L$ by a map of homotopy class $\beta$. Let $x, y, z$ denote the cohomology classes of $K$ which are carried by $e^{n}, S^{q}, e^{n+q}$, respectively. We have to prove that $m$ is the characteristic of $K$, i.e. that

$$
x \cup y=m z \text {. }
$$

Recall that $M=S^{n} \cup L$, and that $g$ maps $S^{q}$ identically and maps $\bar{e}^{n}$ onto both $S^{n}$ and $\bar{e}^{n}$ with degree 1 . Let $N$ denote the complex which is obtained by attaching an $(n+q)$-cell $c^{n+q}$ to $M$ by a map of homotopy class $g_{*}(\beta)$. Let $f: K \rightarrow N$ be a map which agrees with $g$ on $L$ and which maps $e^{n+q}$ onto $c^{n+q}$ with degree 1 . We can think of $N$ as the complex which is obtained from $K$ by identifying to a point the boundary of an $n$-element in the interior of $e^{n}$, so that $f$ is the identi- 
fication map. Consider the induced homomorphism $f^{*}: H^{r}(N) \rightarrow H^{r}(K)$. Let $a^{\prime}, b^{\prime}, c^{\prime}$ denote the cohomology classes of $N$ which are carried by $S^{n}, S^{q}$ and $c^{n+q}$, respectively. Then

$$
f^{*}\left(a^{\prime}\right)=x, \quad f^{*}\left(b^{\prime}\right)=y, \quad f^{*}\left(c^{\prime}\right)=z .
$$

Let $h$ denote the integer-valued homomorphism of $\pi_{n+q-1}(M)$ which is defined in $\$ 4$, with respect to the pair of cohomology classes which are carried by $S^{n}, S^{q}$, respectively. Then $a^{\prime} \cup b^{\prime}=k c^{\prime}$, where $k=h g_{*}(\beta)$, and therefore

$$
\begin{aligned}
x \cup y & =f^{*}\left(a^{\prime}\right) \cup f^{*}\left(b^{\prime}\right)=f^{*}\left(a^{\prime} \cup b^{\prime}\right), \text { by naturality, } \\
& =k f^{*}\left(c^{\prime}\right)=k z .
\end{aligned}
$$

Hence (6.2) is equivalent to the proposition:

$$
h g_{*}(\beta)=m \text {. }
$$

To prove (6.3), we need the following lemma. Let $\kappa_{r} \in \pi_{r}(M)$ $(r=n, q)$ denote the homotopy class of the inclusion map $S^{r} \rightarrow M$. Then $\pi_{n+q-1}(M)$ contains the elements $\kappa_{n} \circ \rho$ and $w=\left[\kappa_{n}, \kappa_{q}\right]$, as in (5.4). Let $u_{*}: \pi_{r}(L) \rightarrow \pi_{r}(M)$ denote the injection. I assert that:

$$
\text { (a) }\left\{\begin{aligned}
h u_{*}(\beta) & =0, \quad h\left(\kappa_{n} \circ \rho\right)=0 ; \\
h(w) & =1 .
\end{aligned}\right.
$$

For consider the complex $S^{n} \cup K$, where $S^{n} \cap K=x_{0}$, which is obtained by attaching $e^{n+q}$ to $M$ by a map of homotopy class $u_{*}(\beta)$. Let $a^{\prime}, b^{\prime}$ denote the cohomology classes of $S^{n} \cup K$ which are carried by $S^{n}, S^{q}$, respectively. Then $a^{\prime} \cup b^{\prime}=0$, since $K$ is a retract of $S^{n} \cup K$, and hence $h u_{*}(\beta)=0$, as asserted. Similarly $h\left(\kappa_{n} \circ \rho\right)=0$, which completes the proof of (a). In the case of (b), let $P$ denote the product complex $S^{n} \times S^{q}$. Let $P^{*}=e^{n} \cup P$, a complex which may be obtained by attaching $c^{n+q}$ to $M$ by a map of homotopy class $w$. Let $a^{\prime}, b^{\prime}, c^{\prime}$ denote the cohomology classes of $P$ which are carried by $S^{n}, S^{q}, c^{n+q}$, respectively, and let $a^{\prime \prime}, b^{\prime \prime}, c^{\prime \prime}$ denote the corresponding classes of $P^{*}$. Then $a^{\prime} \cup b^{\prime}=c^{\prime}$, and hence $a^{\prime \prime} \cup b^{\prime \prime}=c^{\prime \prime}$, i.e. $h(w)=1$. This completes the proof of (6.4).

To return to (6.3), we have

$$
\begin{aligned}
h g_{*}(\beta) & =h\left(u_{*}(\beta)+m w+\kappa_{n} \circ \rho\right), \text { by }(5.4), \\
& =h u_{*}(\beta)+m h(w)+h\left(\kappa_{n} \circ \rho\right), \text { by }(4.1), \\
& =m, \text { by }(6.4) .
\end{aligned}
$$

This proves (6.3), i.e. (3.3), and the proof of (1.2) is now complete. 


\section{REFERENCES}

1. J. Adem, The iteration of the Steenrod squares in algebraic topology, Proc. Nat. Acad. Sci. U.S.A. vol. 38 (1952) pp. 720-726.

2. - Relations on iterated reduced powers, Proc. Nat. Acad. Sci. U.S.A. vol. 39 (1953) pp. 636-638.

3. A. L. Blakers and W. S. Massey, Products in homotopy theory, Ann. of Math. vol. 58 (1953) pp. 295-324.

4. P. J. Hilton, $A$ certain triple Whitehead product, Proc. Cambridge Philos. Soc. vol. 50 (1954) pp. 189-197.

5. P. J. Hilton and J. H. C. Whitehead, Note on the Whitehead product, Ann. of Math. vol. 58 (1953) pp. 429-442.

6. I. M. James, On the homotopy groups of certain pairs and triads, Quart. J. Math. Oxford Ser. (2) vol. 5 (1954) pp. 260-270.

7. - On the suspension sequence, to appear in Ann. of Math.

8. - Multiplication on spheres (II), Trans. Amer. Math. Soc. vol. 84 (1957) pp. $545-558$.

9. W. S. Massey, Exact couples in algebraic topology (Parts I and II), Ann. of Math. vol. 56 (1952) pp. 363-396.

10. N. Nakaoka and H. Toda, On Jacobi identity for Whitehead products, Jour. Inst. Poly. Osaka City Univ. vol. 5 (1954) pp. 1-13.

11. J.-P. Serre, Homologie singuliere des espaces fibres, Ann. of Math. vol. 54 (1951) pp. 425-504.

12. N. E. Steenrod, Cohomology invariants of mappings, Ann. of Math. vol. 50 (1949) pp. 954-988.

13. - Cyclic reduced powers of cohomology classes, Proc. Nat. Acad. Sci. U.S.A. vol. 39 (1953) pp. 217-223.

14. J. H. C. Whitehead, On adding relations to homotopy groups, Ann. of Math. vol. 42 (1941) pp. 409-428.

INSTITUTE fOR Advanced StUdy 